\title{
In Spinal Cord this month . . .
}

The clinical case for this month: respiratory management of a patient with high level tetraplegia

The series of 'Clinical Case of the Month' has in 1997 been under the skilled aegis of Dr William Donovan. In 1998, Dr Fin Biering-Sørenson will be the organiser and director.

In this issue of the journal Menter and his three neurorespiratory experts (p. 805) present and discuss the not uncommon practical clinical-laboratory respiratory problems which occurred in a young man who developed a high level tetraplegia from a c3/4 fracture-dislocation as the result of a skiing accident. The history of the case is given and the questions posed by Menter are mainly related to the subsequent weaning of the patient from the ventilator. Each expert in his own way, brings out relevant points concerning management and Menter considers these and adds his own further comments and recommendations. The difficulty of anticipating the course of the pure respiratory and also of respiratory related events in such patients is discussed, with allusion to prevention of the problems. Menter also provides a useful bibliography.

\section{The causes and costs of spinal cord injuries in the United States}

DeVivo (p. 809) presents a factual, dramatic article on the aetiology and the costs of traumatic SCI in the United States. The economics involved amount to dealing with some 10000 persons a year, and his crosssectional multicentre study brings out many interesting factors, not least that the total direct costs for all causes of SCI in the United States are 7.736 billion US dollars. DeVivo concludes his paper with a strong plea for the prevention of such injuries.

\section{Pain following spinal cord injury}

The types and prevalence of acute and of chronic pain resulting from SCI, and the relationship with psychological well-being are discussed by Kennedy et al (p. 814). They state that the results of their study challenges the belief that pain complaining is solely the manifestation of underlying psychological or adjustment difficulties. Further studies are being done regarding this common and important problem.

\section{The quality of life (QOL) after spinal cord injury}

Intense, important, and most interesting studies are being carried out on the QOL of people who have sustained a severe traumatic SCI, and in this issue of the journal there are three reports, by McColl et al (p. 818); Dijkers (p. 829); and Lin et al (p. 841). Each group has analysed a cohort of patients: McColl et al studied 606 individuals between 1945 and 1990; Dijkers abstracted information from 22 studies; and Lin et al assessed 347 people with a mean age of $37.5 \pm 6.8$ years. A clearer picture of the expectations of life and of health is now becoming available, but it appears that more research is required on what is in fact meant by 'Quality of Life', considered in the general population, and separately for those who have had SCI in particular, as the QOL may or will change sometime following the injury, depending on a number of factors. McColl et al stress that the age at injury is a very important factor, and like Lin, also stress the importance of the level and completeness of the spinal cord lesion. Certainly improvements in the acute care and in rehabilitation are of great importance. Survival after SCI is certainly improving, as is brought out in the data provided in these articles. McColl et al state that their study predicted a medium survival time of 38 years post-injury, with $43 \%$ surviving at least 40 years. A number of information methods are available for these types of study of QOL; McColl et al used the prevalence life table method. Dijkers concentrated on the subjective aspects of QOL, and employed a meta analysis. Lin et al considered five aspects of QOL; physical mobility, environment and transportation, psychosocial adjustment, education, and economics. Both McColl et al and Dijkers agree that an integrated longitudinal reserved view is required for such studies, and Dijkers added that they should utilize clinicalmetrically strong measures of impairment. The question is asked: what do people mean by QOL? As instanced by Dijkers: material possessions, body functions, interpersonal relations, life satisfaction and mental and spiritual well-being. He concludes by saying that with appropriate knowledge about QOL of SCI persons, policy makers may be assisted in deciding what measures should be taken to improve it.

\section{A new disability scale for SCI patients}

The Spinal Cord Independence Measure (SCIM) is a new scale which has been introduced by Catz et al (p. 850) to identify and assess function in spinal paralysed individuals. The authors provide details, and claim that it is more sensitive than the Functional Independence Measure (FIM). The SCIM was administered by a multidisciplinary team. 
Hyperextension injury of the cervical spine

The pathology of a 61 year old man who became tetraplegic from a hyperextension neck injury is described and illustrated by Kinoshita (p. 857).
$\mathrm{X}$-rays did not reveal a fracture or a disc injury. He died 17 months later, and at autopsy there was some extradural haemorrhage, but the main finding was a $5 \mathrm{~mm}$ osteophyte behind the body of $\mathrm{C} 4$ vertebra.

Phillip Harris Editor 